\title{
Influence of Interactive Questions on the Sense of Presence and Anxiety in a Virtual-reality Job-interview Simulation
}

\author{
Shotaro Shimizu \\ Waseda University \\ 2-579-15, Mikajima, Tokorozawa-shi \\ Saitama, Japan. \\ +81-80-6543-0677 \\ c3z-s.wsd@moegi.waseda.jp
}

\author{
Nobuyuki Jincho \\ Waseda University \\ 2-579-15, Mikajima, Tokorozawa-shi \\ Saitama, Japan. \\ +81-4-2947-6985 \\ jincho@aoni.waseda.jp
}

\author{
Hideaki Kikuchi \\ Waseda University \\ 2-579-15, Mikajima, Tokorozawa-shi \\ Saitama, Japan. \\ +81-4-2947-6843 \\ kikuchi@waseda.jp
}

\begin{abstract}
Job interviews are one of the most common methods of selecting employees. However, most people experience a certain amount of anxiety during a job interview. This study aims to help people overcome this anxiety by using a virtual reality job-interview simulation system. This system utilizes voice recognition, a dialogue knowledge base, and morphological analy sis to generate new questions that include nouns from the user's answers.

We expect the user's sense of presence and anxiety to increase with interactive questions. This research used self-assessment questionnaires and psychophysiological measures to investigate two study conditions: (a) using developing questions that included part of the user's answer $(n=10)$ and (b) using a fixed developing question $(n=10)$. The results suggested that using developing questions that included the user's answer provided a greater sense of presence and anxiety.
\end{abstract}

\section{CCS Concepts}

- Human-centered computing $\rightarrow$ Virtual reality

\section{Keywords}

Virtual reality; job interview; interactive question generation; sense of presence; anxiety; biological signal analy sis.

\section{INTRODUCTION}

Job interviews are one of the most common elements in recruitment and entrance examinations. Unfortunately, according to a survey conducted in Japan, more than $60 \%$ of university students are concerned about iob interviews [11. The students' concerns about interviews may be partly due to their low selfefficacy. Self-efficacy [2] refers to the recognition that a person can effectively carry out necessary actions in a certain situation [3] and allows people to change their unadaptable emotions and

\footnotetext{
Permission to make digital or hard copies of all or part of this work for personal or classroom use is granted without fee provided that copies are not made or distributed for profit or commercial advantage and that copies bear this notice and the full citation on the first page. Copyrights for components of this work owned by others than the author(s) must be honored. Abstracting with credit is permitted. To copy otherwise, or republish, to post on servers or to redistribute to lists, requires prior specific permission and/or a fee. Request permissions from Permissions@acm.org.

ICVARS '19, February 23-25, 2019, Perth, WN, Australia (C) 2019 Copyright is held by the owner/author(s). Publication rights licensed to ACM.

ACM 978-1-4503-6592-5/19/02 . \$ $\$ 15.00$
}

DOI: https//doi.org/10.1145/3332305.3332307 behavior [4].

Self-efficacv does not occur spontaneouslv. The most effective way of developing a strong sense of efficacy is through experience [5]. Therefore, it is important to develop high selfefficacy in a job interview thorough experience.

Previous studies $\lceil 6,7\rceil$ implemented job interview simulation svstems in virtual environments and tested their effectiveness based on various aspects of user emotions. For example, the participants who were exposed to a virtual job interview in a fully immersive environment (wearing a head-mounted display) showed a higher level of physiological arousal and self-perceived anxietv than participants in other conditions (42-inch LCD TV. 14-inch laptop screen, audio only ) [7]. Thus, virtual environments are thought to provide users with a more realistic job-interview simulation than through the use of a large screen or computer display can.

The three ideal requirements of a virtual reality system are (a) three-dimensional space, (b) real-time interaction, and (c) selfprojection [8]. The three-dimensional space is a natural setting for human beings. It seems that the above three requirements must be satisfied to reproduce an environment that resembles that of an actual job interview and enh ances the user's sense of presence and anxiety in virtual environments. However, it is considered that existing job interview simulation systems do not provide sufficiently realistic real-time interactions because (as far as we know) only predetermined questions are presented to the users.

This research aims to assist people in overcoming their anxiety concerning job interviews by using a virtual-reality job-interview simulation system that includes an interactive question generator. This paper reports on the implementation of an interactive question generator and its influence on the sense of presence and anxiety during job interviews in a virtual environment.

\section{RELATED WORK}

\subsection{Virtual Reality Exposure Therapy}

Exposure therapy is known to be an effective behavioral treatment for reducing anxiety that is evoked by a stimulus [9]. Virtualreality exposure therapy (VRET) is a form of exposure therapy that stimulates anxiety within a virtual-reality environment [10]. Exposure therapy using real stimuli is called in-vivo exposure and the exposure therapy using imaginary stimuli is called image exposure. In-vivo exposure is known to be more effective than image exposure [9]. 
Unfortunately, in-vivo exposures can be expensive and timeconsuming, for example treating a flight phobia. Furthermore, in in-vivo exposures, a patient may unexpectedly be exposed to an excessively strong stimulus. VRET is a therapeutic method expected to solve these problems. A job-interview simulation system in a virtual environment can help control a user's anxiety by making the virtual interviewer' $\mathrm{s}$ response positive or negative [11].

\subsection{Job-Interview Simulation System in a Virtual Environment}

Previous studies on job-interview simulation systems have investigated the mental state of users in virtual environments. A finding of a previous studies is that a user's anxiety in a virtual job-interview simulation is similar to that in an actual environment [12]. In addition, even though the virtual interviewer with the most realistic graphic details came closest to mimicking the anxiety levels observed in an actual situation, a virtual interviewer at a lower level of realism (such as a cartoon-like virtual interviewer) also successfully caused a significant degree of anxiety [7]. Furthermore, the pitch and speed of the virtual interviewer's voice influenced the user's anxiety [13].

\section{RESEARCH HYPOTHESES}

Existing job-interview simulation systems do not provide sufficiently realistic real-time interactions because only predetermined questions are presented to the users. Therefore, using questions based on the user's answers are considered to produce a greater sense of presence and anxiety. To confirm this research statement, this research investigates the two research hy potheses below.

RH1: Developing questions using the user's answer will be more likely to provoke anxiety.

RH2: Developing questions using the user's answers will increase the sense of presence.

\section{IMPLEMENTATION}

\subsection{System Overview}

Our job-interview simulation system consists of a virtual environment and dialogue function.

The virtual environment is made with Unity (provided by Unity Technologies), and consists of a virtual meeting room and a virtual interviewer (see Figure 1). The size of the virtual meeting room and its furniture were simulated by referring to the actual dimensions of company meeting rooms. The virtual interviewer always moves subtly and moves his lips when speaking his words.

The dialogue function of this system consists of voice synthesis (VoiceText Web API, provided by HOYA), voice recognition (Windows Speech Recognition, provided by Microsoft), and an interactive question generator (see Section 4.2). The basic procedure of the dialogue function is shown in Figure 2. Firstly, a fixed question such as "Please introduce yourself" is presented to an interviewee (i.e., the user) using voice synthesis (Procedure 1). Secondly, the interviewee answers questions orally. At that time, all speech is recorded and converted to text data using voice recognition (Procedure 2). Then, the interactive question generator generates a developing question (Procedure 3), and it is uttered using voice synthesis. Finally, the user answers the new questions (Procedure 4). In an actual scenario, the system repeats Procedures 1 through 4.

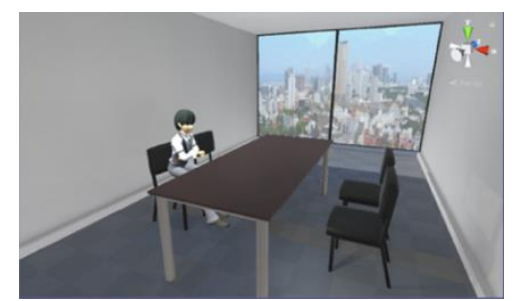

Figure 1. Virtual meeting room and virtual interviewer

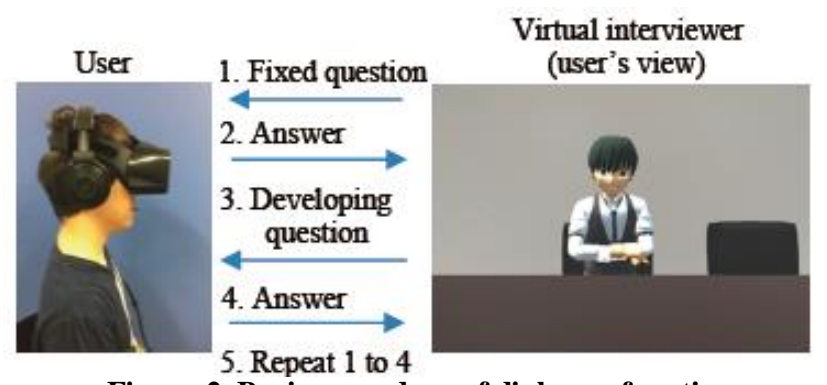

Figure 2. Basic procedure of dialogue function

\subsection{Interactive Question Generator}

The developed interactive question generator (IQG) generates developing questions based on the user's answers. Specifically, IQG first extracts nouns by using a Japanese morphological analyzer (MeCab, available from open source soft ware). Next, the nouns considered to be important in job interviews in Japan are extracted from these. Then, the nouns and five template sentences are combined to generate new questions. Finally, one of the new questions is stochastically chosen as the output.

The list of template sentences (see Table 1) was created with reference to a book containing examples of job-interview questions [14]. IQG has a pre-built knowledge base (see Section 4.3 and Table 3 ) to determine important nouns and combine the nouns and templates.

Table 1. List of template sentences

\begin{tabular}{|l|l|}
\hline 1 & How is [noun] for you? \\
\hline 2 & Are you confident in [noun]? \\
\hline 3 & Why did you choose [noun]? \\
\hline 4 & Please tell me more about [noun]? \\
\hline 5 & What did you learn from [noun]? \\
\hline
\end{tabular}

(Because articles (e.g., a, an, the) are not essential in Japanese, they are not considered.)

\subsection{Knowledge Base}

A total of 143 nouns considered to be important in job interviews in Japan are stored in the knowledge base. The method used to define important nouns is explained below.

First, a questionnaire survey simulating the job applications for 36 university students (each paid 1500 JPY) was conducted. The participants consisted of 14 males and 22 females, with an average age of 20.4 years and a standard deviation (SD) of 2.37. The contents of the questionnaire were the same as the questions used by the virtual interviewer in the system (see Table 2). Each 
question had 300 to 400 characters available for the response.

Next, the frequency with which each noun appeared for each participant was counted. This frequency is called the document frequency (DF), and it is a common indicator of a word's importance. The importance of each noun in the job interview was defined as the DF value of the job applications divided by the DF of Wikipedia. In this process, the DF of general nouns is low and the DF of nouns specific to job interviews is relatively high. The nouns within the top 200 DFs were selected as important terms in job interviews.

Finally, values to express the suitablility of each noun in five templates (see Table 1 in Section 4.2) were obtained by using a cloud-sourcing service. One point was assigned to a noun / template combination evaluated as natural by 15 out of 20 people, and one point was added for each additional person; a maximum of six points could be scored (see Table 3). Nouns not matching any templates were excluded from the list.

The probability that a combination of a certain noun and a template will be chosen as a developing question is weighted by this value. For example, when gakugyo [school work] and daigakusei [university student] are the only important words extracted from the user's answer, the probability that the question, "How is school work for you" will be chosen as the next developing question is $19.3 \%$ (quotient obtained by dividing 6 by $6+3+6+6+6+4+0+0+0+0(31))$.

Table 2. Questions on job application

\begin{tabular}{|c|l}
\hline 1 & Please introduce yourself. \\
\hline 2 & $\begin{array}{l}\text { Please tell me the type of work you want to do, and the reason } \\
\text { for it. }\end{array}$ \\
\hline 3 & What were you most enthusiastic during your school days? \\
\hline 4 & What are your strengths? \\
\hline 5 & What are your weaknesses? \\
\hline 6 & $\begin{array}{l}\text { Please tell us the greatest difficulty you have experienced and } \\
\text { how you overcame it. }\end{array}$ \\
\hline 7 & What kind of person do your coworkers say you are? \\
\hline
\end{tabular}

Table 3. Examples of fitting each noun to the five templates

\begin{tabular}{|c|c|c|c|c|c}
\hline & Template 1 & 2 & 3 & 4 & 5 \\
\hline gakugyo [school work] & 6 & 3 & 6 & 6 & 6 \\
\hline daigakusei [university student] & 4 & 0 & 0 & 0 & 0 \\
\hline puroguramingu [programming] & 3 & 5 & 2 & 3 & 4 \\
\hline arubaito [part time job] & 6 & 0 & 5 & 6 & 6 \\
\hline zemi [seminar] & 3 & 0 & 6 & 6 & 6 \\
\hline
\end{tabular}

\section{METHOD}

\subsection{Experimental Design}

This research aimed to investigate the influence of questions by using the user's answers on the presence and anxiety in a virtualreality job-interview simulation. Therefore, the participants were divided into two groups. One group (Interactive Questions Group) was asked a developing question including a noun from the user's response to a fixed question (see Figure 2 in Section 4.1). The other group (Fixed Questions Group) were asked the same developing question; "Please tell me a bit more," for each answer to a fixed question.

\subsection{Participants}

Twenty-two university students participated in this experiment (each paid $2000 \mathrm{JPY}$ ). The dataset consisted of 11 males and 11 females with an average age of 21.5 years and a standard deviation of 1.07. The participants were evenly divided between the Interactive Questions Group and Fixed Questions Group.

\subsection{Apparatus}

The participants wore a head-mounted display (FOVE 0) with a headphone and microphone, a wireless heart-rate monitor (myBeat-WHS-1), and an electrodermal activity monitor (APU030).

\subsection{Data Acquisition}

\subsubsection{Self-assessment questionnaires}

All participants completed a questionnaire on the Short Fear of Negative Evaluation Scale (SFNE) [15] before the job interview. SFNE is commonly used to assess people's fear of a negative evaluation. After the job interview, the participants answered a 32-items presence questionnaire (PQ) [16], which asked them to rate their experience on a five-point scale to measure the sense of presence experienced in the virtual environment.

However, five questions (Q.17, 21, 24, 27 and 29) were excluded because they did not apply to the system. For example, "Q.17 How well could you actively survey or search the virtual environment using touch?" could not be answered because the system did not use touch.

\subsubsection{Physiological measurement}

Physiological measurements related to anxiety comprised the R-R interval (RRI), skin-conductance response (SCR), and eye blinks (EBs). The RRI was measured using a heart-rate monitor. The SCR was measured using an electrodermal activity monitor. EBs were measured using the head-mounted display.

\subsubsection{User profile}

Personal differences may sometimes affect the data. Therefore, a profile for each user, including their age, sex, and job-interview experience was created.

\subsection{Procedure}

The study protocol received approval from the ethics committee of Waseda University. The participants provided written informed consent prior to their participation. Then. thev completed the SFNE. After that, they put on the head-mounted display with a headphone and microphone, the wireless heart-rate monitor, and the electrodermal activity monitor. Before the job interview, psy chophy siological data were collected for five minutes was as a baseline. The baseline data were measured as the participants watched scenes without any sounds or object movements (see Figure 3).

In the job interview, all participants were asked the seven fixed questions from the job application (see Table 2 in Section 4.3). The time at which a question ended was determined bv the person conducting the experiment. After each fixed question, the participants of the Interactive Questions Group were asked a developing question including a noun from their answer. If no important nouns were used, they were asked the developing question, "Please tell me a bit more." However, our system was able to extract important nouns from roughly $80 \%$ of all fixed 
questions. The participants of the Fixed Questions Group were always asked the same developing question, "Please tell me a bit more."

Following the job interview, all participants completed the PQ. Finally, they received an explanation of this study's experimental design and their payment.

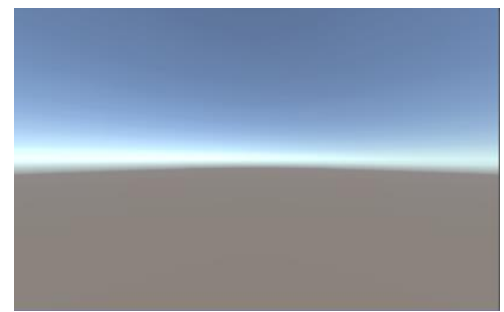

Figure 3. Scene for measuring the baseline psychophysiological data

\section{RESULTS}

The difference in the anxiety of participants from the mean RRI change was examined between the Interactive Questions Group and the Fixed Questions Group. The mean RRI change was obtained by subtracting the mean RRI during the job interview from the baseline RRI. Prior to the analy sis, data whose mean RRI change was over two SDs from the mean were excluded as outliers; each group had 10 data points.

Next, a regression model was constructed to investigate whether there was a significant difference in the mean RRI change. The constructed multiple regression model consisted of the mean RRI change as the dependent variable, and group, sex, and age as the explanatory variables (see Table 4). To compare the influence among the variables, group and sex were used as dummy variables (Group Interactive Questions Group $=1$, Fixed Questions Group $=0$, male $=1$, and female $=0$ ) and all variables were standardized.

The results confirmed a significant effect of Group, Sex, and Age. The mean RRI change of the participants in the Interactive Questions Group was smaller than that of the participants in the Fixed Questions Group. In addition, the female participants had a smaller mean RRI change than the male participants, and the younger participants had a smaller mean RRI change than the older ones. The estimated effect of group was slightly greater than the effects of sex and age.

Table 4. Multiple regression model explaining mean RRI change

\begin{tabular}{|l|c|c|c|c|c}
\hline \multicolumn{9}{|c}{$(* \mathrm{p}<0.05, * * \mathrm{p}<0.01, * * * \mathrm{p}<0.001)$} \\
\hline & Estimate & Std. Error & $\mathrm{t}$ value & $\mathrm{p}$ value & Sig. \\
\hline Intercept & -79.315 & 8.659 & -9.159 & $<.01$ & $* * *$ \\
\hline (scale) Group & -24.812 & 9.012 & -2.753 & .014 & $*$ \\
\hline (scale) Sex & 21.008 & 8.930 & 2.352 & .032 & $*$ \\
\hline (scale) Age & 20.792 & 8.966 & 2.319 & .034 & $*$ \\
\hline
\end{tabular}

\section{DISCUSSION}

RH1. The participants of the Interactive Questions Group had a smaller mean RRI change than those in the Fixed Questions Group. Generally, heart rate increases during an xiety. Conversely, RRI becomes smaller when during anxiety because it denotes the interval between heartbeats. Therefore, developing questions using the user's answers were more likely to provoke anxiety. The reason for this result is considered to be the participants had a stronger sense that the interviewer was listening to them.

Furthermore, the female participants had a smaller mean RRI change than that of the male participants. This may possibly be because the interviewer was male. In addition, the younger participants had a smaller mean RRI change. This may be because older people generally have more experience of communicational situations, including job interviews. The estimated effect of group was slightly greater than the effects of sex and age.

RH2. In the case where no presence cloud be obtained, it was considered that no anxiety occurred because the user's experience deviated from that of an actual interview. Participants in the Interactive Questions Group felt more anxious than those in the Fixed Questions Group. Thus, they should feel a sense of presence with anxiety. Detailed analysis of the PQ dataset must be conducted to consider the degree of the user's presence.

For future work, additional analysis of the SCR, EB, and PQ datasets needs to be conducted to increase the reliability of this result.

\section{CONCLUSION}

This research developed a virtual-reality job-interview simulation system that could interactively generate developing questions including a noun from the user's answer. The participants who experienced developing questions using the user's prior answers had a smaller mean RRI change than those who experienced fixed questions. Therefore, developing questions using the user's answers is more likely to provoke anxiety and make users feel a sense of presence.

However, this question generator does not consider the context of a whole conversation. Generated questions that consider the context are expected in future studies. In addition, the proposed system generates developing questions stochastically. However, patients undergoing exposure therapy experience weak stimuli during the early stages of treatment and gradually get accustomed to stronger stimuli. Likewise, implementation of a functionality that focuses on gradually evoking a strong sense of anxiety is expected. This functionality is to be realized through the distinction between a developing question tends to increase anxiety or not.

\section{REFERENCES}

[1] License Academy. 2014. Shushoku ni kansuru ishikichosa [Survey of thinking on employment]. Retrieved September 25, 2018. Web site: https://licenseacademy.jp/webroot/pdf/industry/pdf_indu_cen t_140718.pdf (in Japanese)

[2] Bandura, A. 1977. Self-efficacy: Toward a unifying theory of behavioral change. Psychological Review. 84, 191-215.

[3] Narita, K., Shimonaka, Y., Nakazato, K., Kawaai, C., Sato, S., and Osada, Y. 1995. A JAPANESE VERSION OF THE GENERALIZED SELF-EFFICACY SCALE - Scale utility from the life-span perspective. Japanese Journal of Educational Psychology. 43, 306-314.

[4] Sakano, Y. and Tohjoh, M. 1986. THEGENERAL SELFEFFICACY SCALE (GSES): SCALE DEVELOPMENT 
AND VALIDATION. Jap. J. Ther., 12, 1, 73-82.

[5] Bandura, A. 1994. Self-efficacy. Encyclopedia of Human Behavior. 4, 71-81.

[6] VIRTUAL SPEECH. (n.d.). Job Interview Preparation, Retrieved September 25, 2018. Web site: https://virtualspeech.com/courses/job-interview.

[7] Kwon, J. H., Powell, J., and Chalmers, A. 2013. How level of realism influences anxiety in virtual reality environments for a job interview. Int. J. Human-Computer Studies. 71, 979-987.

[8] Tachi, S., Sato, M., and Hirose, M. 2011. Virtual Reality. The Virtual Reality Society of Japan.

[9] Marks, I. M. 1987. Fears, phobias and rituals: Panic, anxiety and their disorders. Oxford University Press, New York.

[10] Miyano, M. 2015. Virtual Reality Exposure Therapy using $360^{\circ}$ Panoramic Movies: A Case Study of Acrophobia. Japanese Journal of Medical Virtual Reality. 13, 1, 1-10.

[11] Hartanto, D., Kampmann, I. L., Morina, N., Emmelkamp, P. G. M., Neerincx., M. A., and Brinkman, W. 2014. Controlling Social Stress in Virtual Reality Environments. PLOS ONE. March 2014, 9, 3.
[12] Villani, D., Reppeto, C., Gipresso, P., and Riva, G. 2012. May I experience more presence in doing same thing in virtual reality than in reality? An answer from a simulated job interview. Interacting with Computer. 4, 265-272.

[13] Shimizu, S., Liao, J. A., Jincho, N., and Kikuchi, H. 2017. Evaluation of anxiety using pulse rate variability associated with speaking rate and pitch of the VR-simulated interviews. Forum on Information Technology. 16, 3, 139-142.

[14] Sugimura, T. and Kumagai, T. 2017. Zettai naitei 2019 mensetsu no shitumon [Absolutely job offer acquisition 2019, job interview question]. Daiy amondo sha [Diamond Press]. (in Japanese)

[15] Sasagawa, S., Kanai, Y., Muranaka, Y., Suzuki, S., Shimada, H. and Sakano, Y. 2004. Development of a Short Fear of Negative Evaluation Scale for Japanese Using Item Response Theory. Japanese Journal of Behavior Therapy. 30, 2, 87-98.

[16] Witmer, G, B. and Singer, J, M. 1998. Measuring Presence in Virtual Environments: A Presence Questionnaire. Presence: Teleoperators and Virtual Environments. 7, 3, 225-240. 